\title{
QCD at zero baryon density and the Polyakov loop paradox
}

\author{
Slavo Kratochvila ${ }^{1, *}$ and Philippe de Forcrand ${ }^{1,2, \dagger}$ \\ ${ }^{1}$ Institute for Theoretical Physics, ETH Zürich, CH-8093 Zürich, Switzerland \\ ${ }^{2}$ CERN, Physics Department, TH Unit, CH-1211 Geneva 23, Switzerland
}

(Received 23 February 2006; published 28 June 2006)

\begin{abstract}
We compare the grand-canonical partition function at fixed chemical potential $\mu$ with the canonical partition function at fixed baryon number $B$, formally and by numerical simulations at $\mu=0$ and $B=0$ with four flavors of staggered quarks. We verify that the free energy densities are equal in the thermodynamic limit, and show that they can be well described by the hadron resonance gas at $T<T_{c}$ and by the free fermion gas at $T>T_{c}$. Small differences between the two ensembles, for thermodynamic observables characterizing the deconfinement phase transition, vanish with increasing lattice size. These differences are solely caused by contributions of nonzero baryon density sectors, which are exponentially suppressed with increasing volume. The Polyakov loop shows a different behavior: for all temperatures and volumes, its expectation value is exactly zero in the canonical formulation, whereas it is always nonzero in the commonly used grand-canonical formulation. We clarify this paradoxical difference, and show that the nonvanishing Polyakov loop expectation value is due to contributions of nonzero triality states, which are not physical, because they give zero contribution to the partition function.
\end{abstract}

DOI: 10.1103/PhysRevD.73.114512

PACS numbers: 11.15.Ha, $12.38 . \mathrm{Gc}$

\section{INTRODUCTION}

To simulate QCD thermodynamics on the lattice [1], one commonly uses the grand-canonical partition function with respect to the quark number as a function of a chemical potential $\mu$. It is given, after integration over the fermion fields, by

$$
Z_{\mathrm{GC}}(T, \mu)=\int[D U] e^{-S_{g}[\beta, U]} \operatorname{det} M(U ; \mu) .
$$

Recently, another approach using a canonical formalism has been used [2,3]. ${ }^{1}$ The canonical partition function, which will be derived in detail in the next section, is

$$
Z_{C}(T, Q)=\int_{-\infty}^{\infty} d\left(\frac{\mu_{I}}{T}\right) e^{-i Q \mu_{I} / T} Z_{\mathrm{GC}}\left(T, \mu=i \mu_{I}\right) .
$$

The physics described by both ensembles, grand canonical and canonical, must be the same in the thermodynamic limit, i.e. the free energy density should be the same. This has been shown in Refs. [5,6], and will be confirmed in this study. It is thus puzzling that the expectation value of the Polyakov loop (the world line of a static charge) is exactly zero in the canonical ensemble, while it is nonzero in the grand-canonical ensemble, for all temperatures and volumes. We will show that this discrepancy is due to contributions from the canonical sectors with a quark number

\footnotetext{
*Electronic address: skratoch@itp.phys.ethz.ch

${ }^{\dagger}$ Electronic address: forcrand@itp.phys.ethz.ch

${ }^{1}$ We should mention that a study of QCD thermodynamics in the canonical ensemble was performed already several years ago [4], in the heavy mass (quenched) limit. The action in Ref. [4] is manifestly $Z_{3}$ symmetric and the Polyakov loop expectation value vanishes identically. However, the expectation value of the magnitude of the Polyakov loop can be used to distinguish the low- and high-temperature phases.
}

that is not a multiple of three: the so-called nonzero triality sectors. ${ }^{2}$ Discussions on the role of these nonzero triality sectors have a long history [7] and include speculations that their influence persists even in the thermodynamic limit, so that they must be explicitly projected out. It is our purpose to clarify these issues.

In the following, we discuss properties of the grandcanonical partition function as a function of an imaginary chemical potential and construct the canonical partition function (Sec. II). We then show that the Polyakov loop vanishes in the canonical ensemble (Sec. III), and resolve the paradox above (Sec. IV). After presenting our numerical method to simulate the zero baryon density sector, which is the first step towards finite density QCD simulations (Sec. V), we elaborate on the results for the free energy density as a function of the imaginary chemical potential, which we compare with predictions of the hadron resonance gas model [8] and of a free fermion gas (Sec. VI). We further study the expectation values and finite-size effects of thermodynamic observables, like the plaquette and the chiral condensate in both formulations (Sec. VII). Conclusions follow. Preliminary results of this study have been presented in Ref. [6].

\section{CANONICAL ENSEMBLE}

Let us first discuss symmetries of the grand-canonical partition function $Z_{\mathrm{GC}}(T, \mu)$ as a function of an imaginary chemical potential $\mu=i \mu_{I}$, following Ref. [9]:

(i) Evenness: $Z_{\mathrm{GC}}\left(-i \mu_{I}\right)=Z_{\mathrm{GC}}\left(+i \mu_{I}\right)$. The transformation $\mu \rightarrow-\mu$ can be compensated by time reversal, i.e. by interchanging particles and

\footnotetext{
${ }^{2}$ Triality is defined as the difference between the number of quarks and the number of antiquarks modulo 3 .
} 
antiparticles. Time reversal is equivalent to $C P$ symmetry (since $C P T$ is always a good symmetry), and thus does not change the thermodynamics in the absence of $C P$ violating terms.

(ii) $\frac{2 \pi T}{3}$-periodicity in $\mu_{I}: \quad Z_{\mathrm{GC}}\left(i\left(\mu_{I}+\frac{2 \pi T}{3}\right)\right)=$ $Z_{\mathrm{GC}}\left(i \mu_{I}\right)$. A shift in the imaginary chemical potential $\mu_{I} \rightarrow \mu_{I}+\frac{2 \pi k}{3} T$ can be exactly compensated by a $Z_{3}$ center transformation, also called a nonperiodic gauge transformation $\Omega$, where the temporal gauge links, $U_{4}\left(\vec{x}, x_{4}\right)=\exp \left(\operatorname{igaA}_{4}\left(\vec{x}, x_{4}\right)\right)$, are changed to $\Omega\left(x_{4}\right) U_{4}\left(\vec{x}, x_{4}\right) \Omega^{\dagger}\left(x_{4}+a\right)$, with $\Omega\left(x_{4}+N_{t} a\right)=z(k) \Omega\left(x_{4}\right) \quad\left(N_{t}\right.$ is the number of temporal sites on the lattice and $a$ the lattice spacing) and $z(k) \equiv e^{i(2 \pi k / 3)} \mathbb{1}_{3}$. As a consequence, the Polyakov loop, which is given by $\operatorname{Pol}(\vec{x})=$ $\frac{1}{N_{c}} \operatorname{Tr} \prod_{n_{4}=1}^{N_{t}} U_{4}\left(\vec{x}, x_{4}=n_{4} a\right)$ is also rotated: $\operatorname{Pol}(\vec{x}) \rightarrow e^{-i(2 \pi k / 3)} \operatorname{Pol}(\vec{x})$. However, since the path integral sums over all possible gauge fields, the partition function stays the same.

These properties lead to an expectation for the phase structure in the $T-\mu_{I}$ plane, see Ref. [9] and Fig. 1. The fermion determinant breaks the $Z_{3}$ symmetry explicitly, and the Polyakov loop acquires a nonzero expectation value. The orientation of the Polyakov loop in the complex plane depends on $\mu_{I}$, as indicated in Fig. 1. At high temperature, the free energy density can be obtained as a function of $\mu_{I} / T$ by perturbation theory. It has cusps at $\frac{\mu_{I}}{T}=(2 k+1) \frac{\pi}{3}$. The derivative with respect to $\mu_{I}$ is discontinuous, indicating first-order transitions, during which the Polyakov loop "jumps" from one $Z_{3}$ sector to another. At low temperature, these $Z_{3}$ transitions become crossovers and the vertical, first-order transition lines in Fig. 1

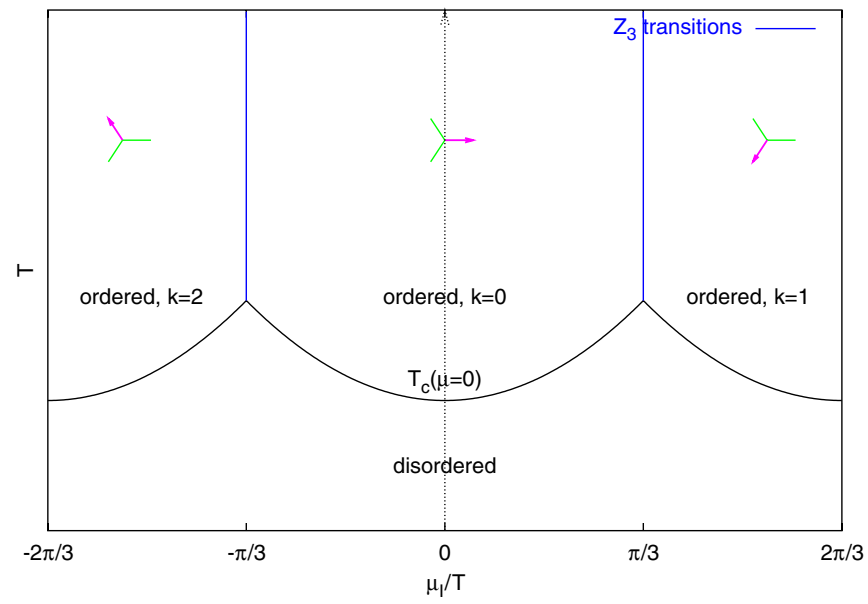

FIG. 1 (color online). Phase diagram of $Z_{\mathrm{GC}}\left(i \mu_{I}\right)$ in the $\left(\mu_{I}\right.$, $T)$ plane. The arrows indicate the orientation of the Polyakov loop. The vertical lines mark the "order-order" $Z_{3}$ transitions, which are first order. Properties (esp. crossover or genuine phase transition) of the "order-disorder" (curved) lines depend on the parameters (number of flavors, quark masses) of the theory. terminate. Whether or not a genuine phase transition separates the high-temperature "ordered" regime and the lowtemperature "disordered" one is a dynamical issue depending on the chosen QCD parameters (number of flavors, quark masses).

To obtain the canonical partition function $Z_{C}(T, Q)$, we fix to $Q$ the conserved charge $\hat{N}=\int d^{3} \vec{x} \bar{\psi}(\vec{x}) \gamma_{0} \psi(\vec{x})$, which represents the net quark number. This is accomplished by inserting a $\delta$-function in the grand-canonical partition function:

$$
\begin{aligned}
Z_{C}(T, Q)= & \int[D U][D \bar{\Psi}][D \Psi] e^{-S_{g}[U ; T]-S_{F}[U, \bar{\Psi}, \Psi ; T]} \\
& \times \delta(\hat{N}-Q) .
\end{aligned}
$$

The $\delta$-function admits a Fourier representation with the new variable $\bar{\mu}_{I}$ :

$$
\begin{aligned}
Z_{C}(T, Q)= & \mathcal{N} \int_{-\infty}^{\infty} d \bar{\mu}_{I} e^{-i Q \bar{\mu}_{I}} \times \int[D U][D \bar{\Psi}] \\
& \times[D \Psi] e^{-S_{g}[U ; \beta]-S_{F}[U, \bar{\psi}, \psi]+i \bar{\mu}_{I} \hat{N}} \\
= & \mathcal{N} \int_{-\infty}^{\infty} d \bar{\mu}_{I} e^{-i Q \bar{\mu}_{I}} \times \int[D U][D \bar{\Psi}][D \Psi] \\
& \times e^{-S_{g}[U ; \beta]-S_{F}[U, \bar{\psi}, \psi]+i \bar{\mu}_{I} \int d^{3} \vec{x} \bar{\psi}(\vec{x}) \gamma_{0} \psi(\vec{x})} \\
= & \mathcal{N} \int_{-\infty}^{\infty} d \bar{\mu}_{I} e^{-i Q \bar{\mu}_{I}} \times \int[D U][D \bar{\Psi}][D \Psi] \\
& \times e^{-S_{g}[U ; \beta]-S_{F}[U, \bar{\psi}, \psi]+i \bar{\mu}_{I} T \int_{0}^{1 / T} d \tau \int d^{3} \vec{x} \bar{\psi}(\vec{x}) \gamma_{0} \psi(\vec{x})} .
\end{aligned}
$$

where $\mathcal{N}$ is a normalization factor. In the last line, we have used the fact that $Q$ is conserved.

One recognizes $i \mu_{I}=i \bar{\mu}_{I} T$ as an imaginary chemical potential, so that

$$
\begin{aligned}
Z_{C}(T, Q) & =\mathcal{N} \int_{-\infty}^{\infty} d \bar{\mu}_{I} e^{-i Q \bar{\mu}_{I}} Z_{\mathrm{GC}}\left(T, i \bar{\mu}_{I} T\right) \\
& =\frac{3}{2 \pi} \int_{-\pi / 3}^{\pi / 3} d \bar{\mu}_{I} e^{-i Q \bar{\mu}_{I}} Z_{\mathrm{GC}}\left(T, i \bar{\mu}_{I} T\right),
\end{aligned}
$$

where we have exploited the $\frac{2 \pi T}{3}$-periodicity in $\mu_{I}$ of $Z_{\mathrm{GC}}\left(i \mu_{I}\right)$ in the last step. ${ }^{3}$ From this periodicity, it follows that $Z_{C}(T, Q)=0$ except for $\frac{Q}{3} \equiv B \in \mathbb{Z}$, where $B$ is the baryon number. The canonical partition function vanishes for noninteger baryon number, i.e. for nonzero triality sectors. Note that our argument does not rely on particular spatial boundary conditions (b.c.). The same conclusion holds for periodic or, for example, $C$-periodic spatial b.c., even though the latter break the $Z(3)$ symmetry. For convenience, we write

\footnotetext{
${ }^{3}$ Note that the evenness of $Z_{\mathrm{GC}}\left(i \mu_{I}\right)$ in $\mu_{I}$ implies $Z_{C}(T, Q)=$ $Z_{C}^{*}(T,-Q)$. In particular the $Z_{C}(T, Q)$ 's are real as expected.
} 


$$
Z_{C}(T, B)=\frac{1}{2 \pi} \int_{-\pi}^{\pi} d\left(\frac{\mu_{I}}{T}\right) e^{-i 3 B \mu_{I} / T} Z_{\mathrm{GC}}\left(T, i \mu_{I}\right) .
$$

Now, the fugacity expansion allows us to go back from the canonical partition functions to the grand-canonical one:

$$
Z_{\mathrm{GC}}(T, \mu)=\sum_{Q=-\infty}^{+\infty} e^{Q \mu / T} Z_{C}(T, Q) .
$$

This expression is identical to Eq. (1), as can be seen by substituting (5) for $Z_{C}(T, Q)$ above and summing over $Q$ first. However, we can remove from the sum the nonzero triality (fractional $B$ ) sectors, since each of them gives a zero contribution, thus obtaining:

$$
Z_{\mathrm{GC}}(T, \mu)=\sum_{B=-\infty}^{\infty} e^{3 B \mu / T} Z_{C}(T, B) .
$$

Let us stress again that this grand-canonical partition function is identical with the one given in Eq. (1). The two expressions differ by the inclusion of nonzero triality sectors, which we just showed are zero. However, these zero contributions are explicitly projected out in (8) [7]. In the following, we will come back to this and consider the effect of this projecting out on the $Z_{3}$-sensitive Polyakov loop.

\section{POLYAKOV LOOP IN THE CANONICAL ENSEMBLE}

The expectation value of the Polyakov loop in the canonical ensemble is zero for all temperatures and volumes. We show this explicitly as follows. The chemical potential is introduced on the lattice as the temporal component of an external imaginary gauge field

$$
\begin{gathered}
U_{4}\left(\vec{x}, x_{4}\right) \rightarrow e^{+\mu a} U_{4}\left(\vec{x}, x_{4}\right), \\
U_{4}^{\dagger}\left(\vec{x}, x_{4}\right) \rightarrow e^{-\mu a} U_{4}^{\dagger}\left(\vec{x}, x_{4}\right),
\end{gathered}
$$

or equivalently as

$$
\begin{gathered}
U_{4}\left(\vec{x}, x_{4}=x_{4_{0}}\right) \rightarrow e^{+N_{t} \mu a} U_{4}\left(\vec{x}, x_{4}=x_{4_{0}}\right), \\
U_{4}^{\dagger}\left(\vec{x}, x_{4}=x_{4_{0}}\right) \rightarrow e^{-N_{t} \mu a} U_{4}^{\dagger}\left(\vec{x}, x_{4}=x_{4_{0}}\right),
\end{gathered}
$$

on a given temporal hyperplane $x_{4_{0}}$. An imaginary chemical potential $i \mu_{I}=i \frac{2 \pi T k}{3}$ can then be absorbed in a $Z_{3}$ center transformation

$$
\begin{aligned}
U_{4}\left(\vec{x}, x_{4}=x_{4_{0}}\right) & \rightarrow e^{i N_{t} a 2 \pi T k / 3} U_{4}\left(\vec{x}, x_{4}\right) \\
& =z(k) U_{4}\left(\vec{x}, x_{4}=x_{4_{0}}\right)
\end{aligned}
$$

with $z(k) \equiv e^{i 2 \pi k / 3} \mathbb{1}_{3}$. As a consequence, the two configurations $\left\{U, \mu_{I}\right\}$ and $\left\{z(k) U, \mu_{I}-\frac{2 \pi T k}{3}\right\}$ have the same value for the Dirac determinant $\operatorname{det} M\left(U ; \mu_{I}\right)=$ $\operatorname{det} M\left(z(k) U ; \mu_{I}-\frac{2 \pi T k}{3}\right)$, but the Polyakov loop is centerrotated. We can then group the configurations of a canonical ensemble in triplets having $Z_{3}$-rotated Polyakov loop:

$$
\begin{aligned}
Z_{C}(T, B)= & \frac{1}{2 \pi} \int_{-\pi}^{\pi} d\left(\frac{\mu_{I}}{T}\right) e^{-i 3 B \mu_{I} / T} \int[D U] e^{-S_{g}[U ; \beta]} \\
& \times \frac{1}{3} \sum_{k=0}^{2} \operatorname{det} M\left(z(k) U_{4}\left(x_{4}=x_{4_{0}}\right), \mu_{I}\right) .
\end{aligned}
$$

The three members of a triplet give identical contributions

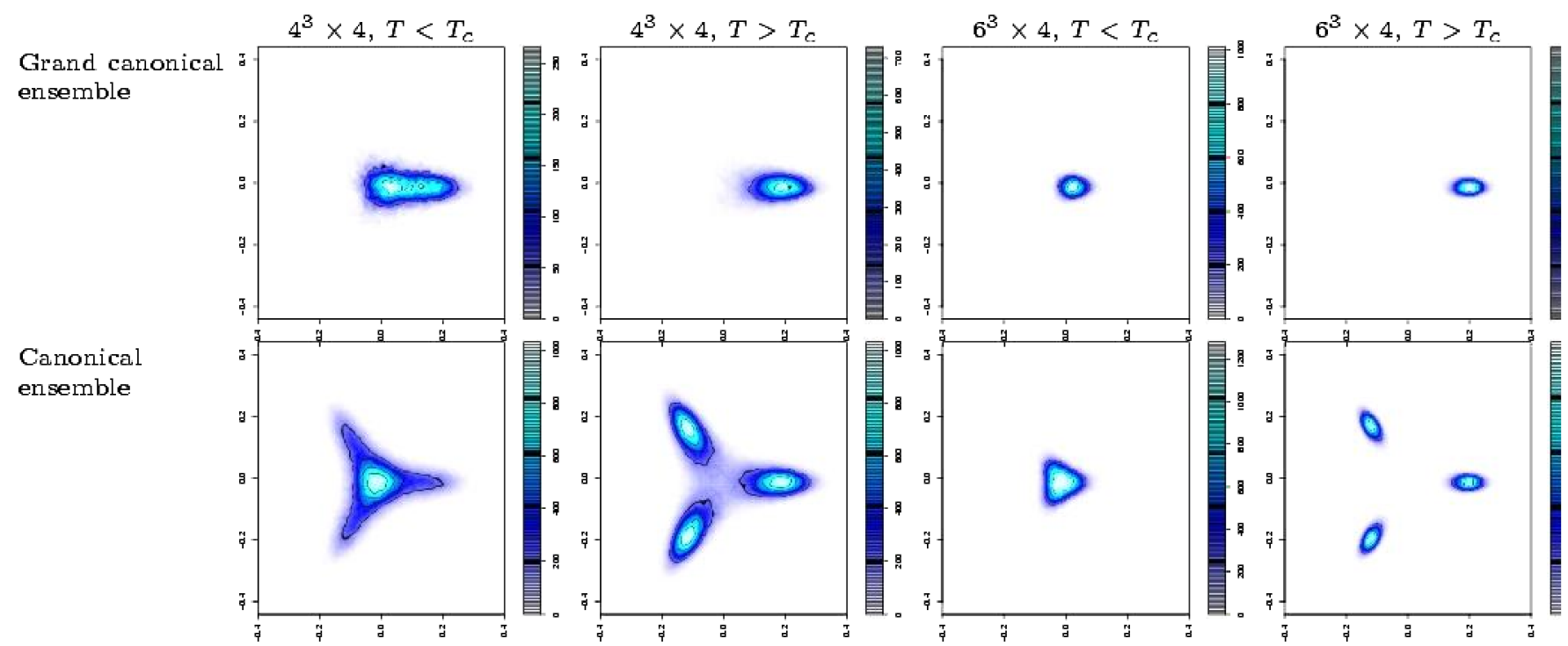

FIG. 2 (color online). Distribution of the complex Polyakov loop trace in the grand-canonical (top) and canonical (bottom) ensembles. Left: $4^{3} \times 4$, right: $6^{3} \times 4$. In the thermodynamic limit, the distributions agree for both ensembles, up to two additional $Z_{3}$-rotations in the canonical ensemble. 
to $Z_{C}(T, B)$, since $\operatorname{det} M\left(z(k) U ; \mu_{I}\right)=\operatorname{det} M\left(U ; \mu_{I}+\frac{2 \pi T k}{3}\right)$ and $e^{-i 3 B\left(\mu_{I} / T\right)(2 \pi T k / 3)}=1$ for $B \in \mathbb{Z}$. In Fig. 2, we show the distribution of the Polyakov loop, where the $Z_{3}$ symmetry (and hence the triplets) is clearly visible in the canonical ensemble (bottom). In each triplet, the average of the Polyakov loops is $\mathrm{Pol}_{i} \times\left(1+e^{-i 2 \pi / 3}+e^{i 2 \pi / 3}\right)=$ 0 , and therefore the ensemble average also vanishes:

$$
\langle\mathrm{Pol}\rangle_{Z_{C}(T, B)}=0
$$

for any integer baryon number and temperature. Note again that the argument does not depend on a particular choice of spatial boundary conditions.

\section{POLYAKOV LOOP IN THE GRAND-CANONICAL ENSEMBLE}

In the ensemble generated by the grand-canonical partition function Eq. (1)

$$
Z_{\mathrm{GC}}(T, \mu)=\int[D U] e^{-S_{g}[U ; \beta]} \operatorname{det} M(U ; \mu),
$$

the fermion determinant explicitly breaks the $Z_{3}$ symmetry, so that the expectation value of the Polyakov loop

$$
\langle\mathrm{Pol}\rangle_{Z_{\mathrm{GC}}(T, \mu)} \neq 0
$$

is nonzero for any chemical potential, temperature, and volume. In the following, we show that this nonvanishing value is caused by canonical sectors with quark numbers which are not a multiple of three.

We express the grand-canonical partition function via the fugacity expansion in the quark number $Q$ (we take $\mu=0$ for notational simplicity only; the argument holds for any $\mu$ ):

$$
\begin{aligned}
Z_{\mathrm{GC}}(T, \mu=0) & =\sum_{Q} Z_{C}(T, Q) \quad \text { with } \quad Z_{C}(T, Q)=0 \quad \text { if } Q \neq 0 \bmod 3 \\
& =\ldots+Z_{C}(0)+Z_{C}(1)+Z_{C}(2)+Z_{C}(3)+Z_{C}(4)+\ldots
\end{aligned}
$$

where $Z_{C}(\cdot)$ indicates $Z_{C}(\cdot)=0$. The canonical partition functions can be written as $Z_{C}(T, Q)=\sum_{i} W_{i}(Q)$, where $i$ labels each configuration, and $W_{i}(Q)$ is the corresponding Boltzmann weight. The expectation value of the Polyakov loop is then generically given by

$$
\langle\mathrm{Pol}\rangle_{\mathrm{GC}}=\frac{\sum_{Q} \operatorname{Num}(Q)}{Z_{\mathrm{GC}}(T, \mu=0)}=\frac{\ldots+\operatorname{Num}(0)+\mathrm{Num}(1)+\mathrm{Num}(2)+\mathrm{Num}(3)+\mathrm{Num}(4)+. .}{\ldots+Z_{C}(0)+Z_{C}(1)+Z_{C}(2)+Z_{C}(3)+Z_{C}(4)+\ldots} \neq 0,
$$

where $\operatorname{Num}(Q)=\sum_{i} \operatorname{Pol}_{i} W_{i}(Q)$, which vanishes if $Q$ is a multiple of 3 due to Eq. (15). It follows that the contributions of canonical sectors with fractional baryon number to the Polyakov loop are unphysical, since the corresponding canonical expectation value is infinite:

$\langle\operatorname{Pol}\rangle_{Z_{C}(T, Q \neq 0 \bmod 3)}=\frac{\operatorname{Num}(Q \neq 0 \bmod 3)}{Z_{C}(Q \neq 0 \bmod 3)}=\frac{\text { nonzero }}{0}=\infty$.

This argument makes it clear that the expectation value of the Polyakov loop is nonzero for all temperatures and volumes, if we use the grand-canonical partition function with respect to the quark number, see Eq. (1) and Fig. 2, top row. However, if we use the grand-canonical partition function with respect to the baryon number, see Eq. (8), the expectation value of the Polyakov loop will be exactly zero even in this equivalent grand-canonical formulation.

Thus, the physical meaning of the $Z_{3}$-sensitive Polyakov loop expectation value is rather limited. It is the
$Z_{3}$-invariant correlator $\left\langle\operatorname{Pol}(0) \operatorname{Pol}(x)^{\dagger}\right\rangle$ which is physical, and indicates confinement or deconfinement by its $|x| \rightarrow$ $\infty$ limit. In the canonical ensemble, this limit is not equal to $|\langle\mathrm{Pol}\rangle|^{2}$, which is identically zero: the clustering property is not satisfied. This is evidence of spontaneous breaking of the center symmetry ${ }^{4}$ in the presence of fermions. The symmetry is broken spontaneously at all temperatures and densities, rather than explicitly as in the usual grandcanonical ensemble.

\section{NUMERICAL APPROACH TO ZERO BARYON DENSITY}

In order to design an algorithm that is able to measure an observable as a function of the quark, or rather baryon number, we need to understand how the expectation value of an observable $\hat{O}$ can be evaluated in the canonical

\footnotetext{
${ }^{4}$ We are grateful to L. Yaffe for pointing this out to us.
} 
ensemble. It is given by

$$
\langle\hat{O}\rangle_{B} \equiv \frac{\frac{1}{2 \pi} \int_{-\pi}^{\pi} d \bar{\mu}_{I} e^{-i 3 B \bar{\mu}_{I}} \int[D U] e^{-S_{g}[U ; \beta]} \operatorname{det} M\left(U ; i \mu_{I}=i \bar{\mu}_{I} T\right) \hat{O}(U)}{Z_{C}(T, B)} .
$$

We recognize the following numerical description. We treat $\bar{\mu}_{I}$ as a dynamical degree of freedom, and supplement the ordinary Monte Carlo (Hybrid MC, R-algorithm, PHMC, RHMC,$\ldots$ ) at fixed $\bar{\mu}_{I}$ with a noisy Metropolis update of $\bar{\mu}_{I} \rightarrow \bar{\mu}_{I}^{\prime}$ keeping the configuration $\{U\}$ fixed. Thus, we alternate two kinds of Metropolis steps.

(i) Update of the links by standard Hybrid Monte Carlo [10]: Keeping the imaginary chemical potential $\mu_{I}$ fixed, we propose a new configuration $\left\{U^{\prime}\right\}$, obtained by leapfrog integration of Hamilton's equations, as a Metropolis candidate. It is then accepted with the ordinary Metropolis probability

$$
\operatorname{Prob}\left(U \rightarrow U^{\prime}\right)=\min \left(1, e^{-\Delta S}\right),
$$

where $\Delta S$ is the difference between the action of $\left\{U^{\prime}\right\}$ and that of $\{U\}$.

(ii) Metropolis update of the imaginary chemical potential by a noisy estimator: Keeping the gauge field configuration $\{U\}$ fixed, we propose a new imaginary chemical potential $\mu_{I}^{\prime}$, obtained from $\mu_{I}$ by a random step drawn from an even distribution. The update is based on the acceptance

$$
\begin{aligned}
& \operatorname{Prob}\left(\mu_{I} \rightarrow \mu_{I}^{\prime}\right) \\
& \quad=\min \left(1, \frac{e^{-i 3 B \mu_{I}^{\prime} / T} \operatorname{det}^{N_{f}}\left(\not D\left(\mu_{I}^{\prime}\right)+m\right)}{e^{-i 3 B \mu_{I} / T} \operatorname{det}^{N_{f}}\left(\not D\left(\mu_{I}\right)+m\right)}\right) .
\end{aligned}
$$

The ratio of determinants is evaluated with a stochastic estimator (see the appendix), namely

$$
\begin{aligned}
& \frac{\operatorname{det}^{N_{f}}\left(\not D\left(\mu_{I}^{\prime}\right)+m\right)}{\operatorname{det}^{N_{f}}\left(\not D\left(\mu_{I}\right)+m\right)} \\
& \quad=\left\langle e^{\left.-\left|\left(\not D\left(\mu_{I}^{\prime}\right)+m\right)^{-N_{f} / 2}\left(\not D\left(\mu_{I}\right)+m\right)^{N_{f} / 2} \eta\right|^{2}+|\eta|^{2}\right\rangle_{\eta},}\right.
\end{aligned}
$$

where $\eta$ is a Gaussian complex vector. Since one Gaussian vector is sufficient, the computational overhead is negligible.

The algorithm above allows the sampling of any positive measure in $\mu_{I}$. However, the oscillatory part $e^{-i 3 B \bar{\mu}_{I}}$ in the sampling weight causes a sign problem for nonzero baryon number $B$. One can use $\left|\cos \left(3 B \bar{\mu}_{I}\right)\right|$ as a sampling measure and fold the remaining sign in the observable. But such an approach breaks down for rather small $B$ already [11]. Nevertheless, at $B=0,\left.e^{-i 3 B \bar{\mu}_{I}}\right|_{B=0}=1$ and thus, the Boltzmann weight is real and positive. Thus, this simple algorithm suits our purpose here. To help ergodicity, we can also perform a " $Z_{3}$-move" at any time:

$$
\begin{aligned}
\mu_{I} & \rightarrow \mu_{I} \pm \frac{2 \pi T}{3}, \\
U_{4}\left(\vec{x}, x_{4}=x_{4_{0}}\right) & \rightarrow U_{4}\left(\vec{x}, x_{4}=x_{4_{0}}\right) e^{\mp i 2 \pi / 3}, \quad \forall \vec{x},
\end{aligned}
$$

where $U_{4}\left(\vec{x}, x_{4}=x_{4_{0}}\right)$ are the temporal links at a given time slice $x_{4_{0}}$. Such a " $Z_{3}$-move" is always accepted, since the configuration $\left\{U, \mu_{I}\right\}$ and the one with a center-rotated Polyakov loop, but shifted imaginary chemical potential, $\left\{U \times e^{-i[(2 \pi) / 3]}, \mu_{I}+\frac{2 \pi T}{3}\right\}$ have the same Dirac determinant, and thus the same sampling weight, as discussed at length in Sec. III.

A computational detail: For $T>T_{c}$, the $\mu_{I}$-distribution is sharply peaked around $0, \pm \frac{2 \pi T}{3}$. To sample this distribution accurately in the whole interval, we apply a multicanonical algorithm in the $T>T_{c}$ regime for the larger lattices $\left(6^{3} \times 4\right.$ and $\left.8^{3} \times 4\right)$ [12]. For this, we bias the sampling of the imaginary chemical potential by modifying the acceptance probability

$$
\begin{aligned}
& \operatorname{Prob}^{\text {multi }}\left(\mu_{I} \rightarrow \mu_{I}^{\prime}\right) \\
& \quad=\min \left(1, \frac{\operatorname{det}^{N_{f}}\left(\not D\left(\mu_{I}^{\prime}\right)+m\right)}{\operatorname{det}^{N_{f}}\left(\not D\left(\mu_{I}\right)+m\right)} e^{\left(\operatorname{bias}\left(\mu_{I}^{\prime}\right)-\operatorname{bias}\left(\mu_{I}\right)\right)}\right),
\end{aligned}
$$

with $\operatorname{bias}\left(\mu_{I}\right)$ chosen such that the sampled histogram becomes flat for all $\mu_{I}{ }^{5}$ The expectation value of an observable $\hat{O}$ is then given by

$$
\langle\hat{O}\rangle=\frac{1}{\sum_{\left\{U ; \mu_{I}\right\}} e^{-\operatorname{bias}\left(U ; \mu_{I}\right)}} \sum_{\left\{U ; \mu_{I}\right\}} \hat{O}\left(U ; \mu_{I}\right) e^{-\operatorname{bias}\left(U ; \mu_{I}\right)},
$$

where $\left\{U ; \mu_{I}\right\}$ labels the configurations $\{U\}$ sampled at imaginary chemical potential $\mu_{I}$.

We focus on four flavors of Kogut-Susskind fermions with degenerate mass $a m=0.05$ and lattices with $N_{t}=4$ time slices, i.e. $\frac{m}{T}=0.2$. With these parameters, the zerotemperature pion mass is about $350 \mathrm{MeV}$ [13]. Simulations are performed on lattices with spatial extents $4^{3}, 6^{3}$, and $8^{3}$ at seven temperatures, ${ }^{6}$ ranging from $\frac{T}{T_{c}}=0.85$ to 1.1 , with good overlap between the "neighboring" ensembles. We analyze the results using Ferrenberg-Swendsen reweighting [14].

\footnotetext{
${ }^{5}$ A simple way to get an estimate of the function bias $\left(\mu_{I}\right)$ is the following: One starts by sampling with no bias to produce a histogram hist $\left(\mu_{I}\right)$ of the sampled $\mu_{I}$. One then fits $\operatorname{bias}\left(\mu_{I}\right)$ to $-\log \left(\operatorname{hist}\left(\mu_{I}\right)\right)$ with a suitable Ansatz like $a \mu_{I}^{2}-b \mu_{I}^{4}$, or uses a table.

${ }^{6} \mathrm{We}$ relate the coupling $\beta$ to the temperature $T$ via $T=\frac{1}{a(\beta) N_{t}}$ and the perturbative two-loop $\beta$-function.
} 


\section{FREE ENERGY DENSITY}

In the grand-canonical ensemble, the change of the free energy density with chemical potential $\mu$ (as a dimensionless quantity) is given in terms of the partition function

$$
\frac{\Delta F(T, \mu)}{V T^{4}} \equiv-\frac{1}{V T^{3}} \log \frac{Z_{\mathrm{GC}}(T, \mu)}{Z_{\mathrm{GC}}(T, 0)} .
$$

A standard approach $[15,16]$ is to perform a Taylor expansion in $\mu$ about $\mu=0$, where the derivatives entering the series may be expressed as complicated expectation values evaluated at $\mu=0$. Remember that this expansion is in even powers of $\mu$, since $Z_{\mathrm{GC}}(\mu)=Z_{\mathrm{GC}}(-\mu)$. In our approach, the free energy density as a function of imaginary chemical potential comes for free from the $\mu_{I}$-histogram in the canonical simulation, and moreover, to all orders. This information can then be used for analytic continuation to real chemical potential.

At low temperature, however, the histograms are quite noisy. Therefore we will, when needed, use results from the more sophisticated method [3], where we can calculate the grand-canonical partition function for an arbitrary imagi- nary chemical potential as a consequence of the reweighting method that we apply.

In Fig. 3 we show the free energy divided by $V T^{4}$ versus $\frac{\mu_{I}}{T}$ for $\frac{T}{T_{c}}<1, \frac{T}{T_{c}} \simeq 1$ and $\frac{T}{T_{c}}>1$. In all cases, we observe a minimum at $\frac{\mu_{I}}{T}=0$. Therefore, in the thermodynamic limit, only $\frac{\mu_{I}}{T}=0 \bmod \frac{2 \pi}{3}$ will survive. This establishes numerically the expected equivalence of $Z_{C}(T, B=0)$ with $Z_{\mathrm{GC}}(T, \mu=0)$.

For $\frac{T}{T_{c}} \sim 0.9$, no singularities develop at $\frac{\mu_{I}}{T}= \pm \frac{\pi}{3}$ in the thermodynamic limit, thus indicating a crossover, as expected from the phase diagram $T$ - $\mu_{I}$, Fig. 1. In Fig. 4 (left), we show the free energy density, determined by the histogram method, which is very flat and noisy unfortunately. The periodicity of the free energy density is $\frac{2 \pi T}{3}$, and we exploit it by a Fourier expansion in $3 k \frac{\mu_{I}}{T}$ using the Ansatz

$$
\frac{\Delta F\left(T, \mu_{I}\right)}{V T^{4}}=c\left(1-\cos \left(3 \frac{\mu_{I}}{T}\right)\right)+d \cos \left(6 \frac{\mu_{I}}{T}\right)+\ldots
$$

In order to improve the determination of the coefficients $c, d, \ldots$, we use results based on the reweighting method
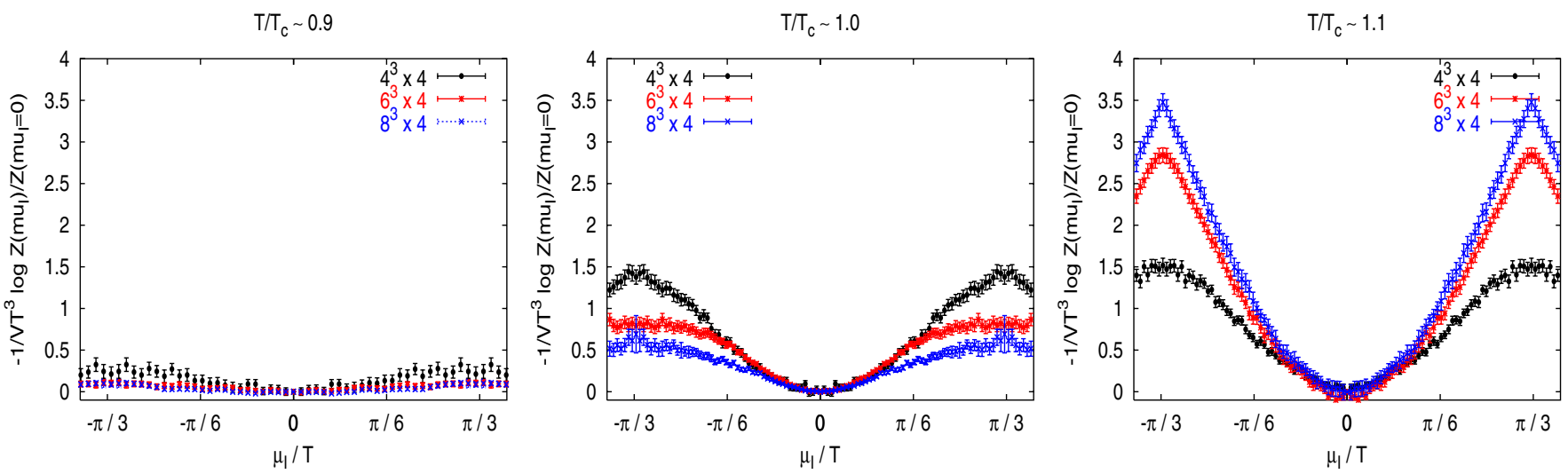

FIG. 3 (color online). $\frac{\Delta F\left(T, \mu_{l}\right)}{V T^{4}}$ as a function of $\frac{\mu_{I}}{T}$, at temperatures $\frac{T}{T_{c}} \sim 0.9,1.0,1.1$ from left to right. The free energy density varies much more upon entering the high-temperature phase, and the $Z_{3}$ first-order transitions become visible (right).
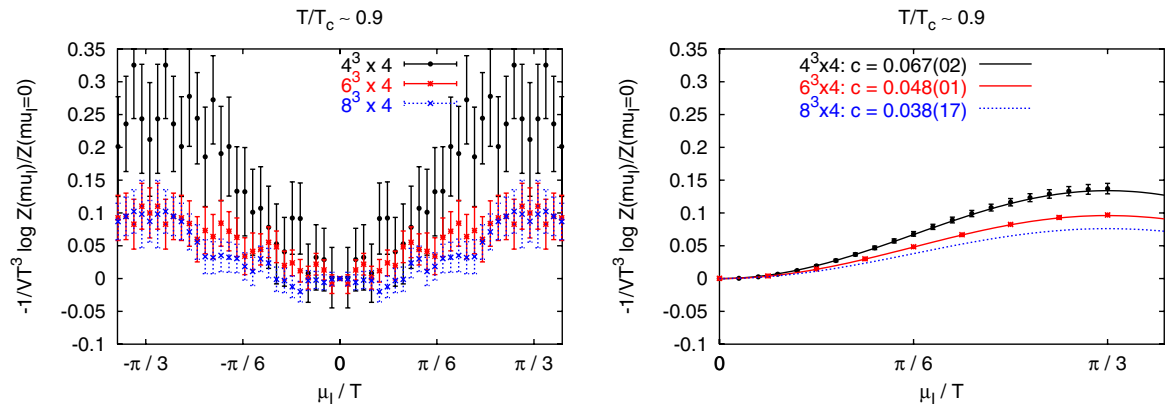

FIG. 4 (color online). $\quad \frac{\Delta F\left(T, \mu_{I}\right)}{V T^{4}}$ as a function of $\frac{\mu_{l}}{T}$ for $\frac{T}{T_{c}} \sim 0.9$. The histogram method is very noisy. We show (left) a rescaled version of the leftmost plot in Fig. 3. We also present (right) results based on a reweighting method with variance reduction [3]. The results are in agreement with the histogram method, but allow for a more reliable description by a Fourier expansion. One Fourier coefficient suffices to describe the data points. The reweighting method [3] calculation is computationally demanding and has not been performed yet for the $8^{3} \times 4$ lattice. We thus only draw the fit, which is based on histogram data. 

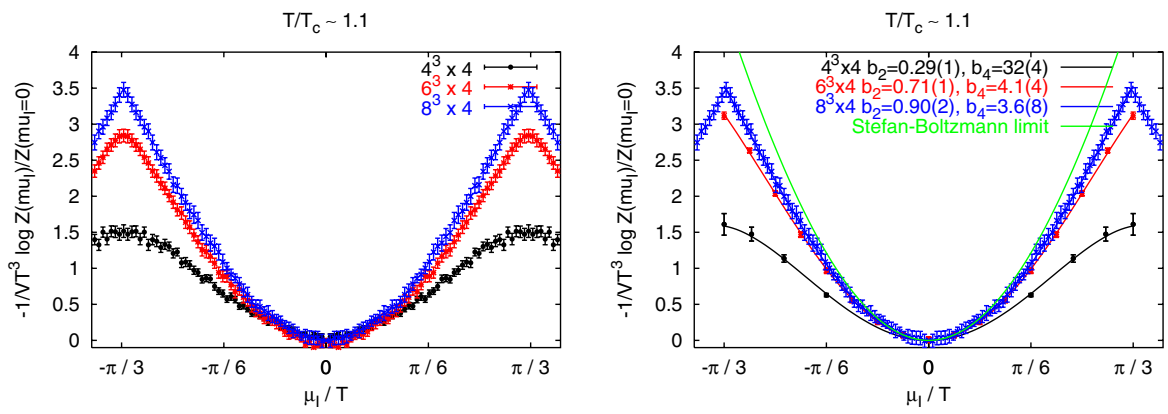

FIG. 5 (color online). $\frac{\Delta F\left(T, \mu_{I}\right)}{V T^{4}}$ as a function of $\frac{\mu_{I}}{T}$ for $\frac{T}{T_{c}} \sim 1.1$. Left: The histogram method, right: the reweighting method [3], supplemented by the histogram results for $8^{3} \times 4$. A simple modification of the free gas expression describes all the data. As the volume increases, the data come close to the Stefan-Boltzmann limit $(T \rightarrow \infty)$ even though $\frac{T}{T_{c}} \sim 1.1$ only.

described in Ref. [3]. Within errors, the free energy density is in agreement with the histogram method, but with much smaller statistical uncertainty. The fit is excellent already with one Fourier coefficient, with no indication for higher Fourier components, at least on the small lattices we consider.

In the hadron resonance gas model (see Ref. [17] for a detailed discussion), the partition function can be split into mesonic and baryonic contributions. Since we are interested in the dependency on a baryon chemical potential $\mu_{B}=3 \mu$, it is sufficient to study the baryonic part only. In the limit $m_{B} \gg T, \mu_{B}$, where $m_{B}$ corresponds to the baryonic resonance mass, the Ansatz for the free energy density as a function of an imaginary chemical potential is

$$
\begin{aligned}
\frac{F\left(T, \mu_{I}\right)}{V T^{4}}-\frac{F(T, 0)}{V T^{4}} & \equiv \frac{\Delta F\left(T, \mu_{I}\right)}{V T^{4}} \\
& =f(T)\left(1-\cos \left(\frac{3 \mu_{I}}{T}\right)\right),
\end{aligned}
$$

where $f(T) \equiv \frac{1}{\pi^{2}} \sum_{i \in \text { Baryons }}\left(\frac{m_{i}}{T}\right)^{2} K_{2}\left(\frac{m_{i}}{T}\right)$. We thus have a mean to measure the sum of resonances $f(T)$. For example in the case of a $6^{3} \times 4$ lattice, we find $f\left(T \sim 0.9 T_{c}\right)=0.048(1){ }^{7}$ Our data can be well described by this Ansatz. This confirms our expectation that the relevant degrees of freedom in the low-temperature phase are hadrons. The masses of these hadrons are much larger than the scale given by the temperature, since the free energy density changes only slightly when varying the imaginary chemical potential, thus $m_{H} \gg \mu_{I} \sim T_{c} \approx$ $160 \mathrm{MeV}$.

For $\frac{T}{T_{c}} \sim 1.1$ we expect a cusp at $\mu_{I}= \pm \frac{\pi T}{3}$ $\left(Z_{3}\right.$-transitions) to develop in the thermodynamic limit,

\footnotetext{
${ }^{7}$ We thank D. Toublan [18] for estimating the sum of resonances for the four-flavor continuum theory. However, the result, $f\left(T \sim 0.9 T_{c}\right) \approx 0.2$, differs from our determination by about a factor 4. It is unclear what is the main reason for this discrepancy, but the small, coarse lattice we use $(a \sim 0.3 \mathrm{fm})$ certainly contributes an important part.
}

due to the first-order phase transition. Indeed, it appears clearly as the volume increases, see Fig. 5 for a comparison of the histogram results (left) versus the reweighting approach $^{8}$ (right).

We can try to describe these results by a generic Taylor series in $\frac{\mu_{I}}{T}$ as an Ansatz, which can be compared with a simple model at high temperature, the free gas of massless quarks. If we perform an analytic continuation from real to imaginary chemical potential, then the free energy density of this model is given by

$$
\frac{\Delta F\left(T, \mu_{I}\right)}{V T^{4}}=\frac{N_{f}}{2}\left(\frac{\mu_{I}}{T}\right)^{2}-\frac{N_{f}}{4 \pi^{2}}\left(\frac{\mu_{I}}{T}\right)^{4} .
$$

These simple expressions are valid in the continuum theory at very high temperature, where the coupling $g(T) \approx 0$. On the lattice we expect finite-size corrections $\left(N_{s}<\infty\right)$ as well as cutoff corrections $\left(T=\frac{1}{a N_{t}}\right)$. Reference [16] has calculated the free energy of free fermions on a lattice having infinite spatial size $\left(N_{s}=\right.$ $\infty)$ but finite temporal extent $\left(N_{t}=4\right)$. Here, we also determine the corrections for finite spatial size $N_{s}=4,6$, 8,10 for the free massless fermion gas on the lattice. We set the gauge fields $A_{\mu}(x)=0$, i.e. the gauge links to the identity, and solve for the free energy via

$$
\begin{aligned}
\frac{\Delta F_{\text {latt }}^{\mathrm{free}}\left(T, \mu_{I}\right)}{V T^{4}} & =-\frac{\log Z^{\mathrm{free}}\left(T, \mu_{I}\right)}{V T^{3}} \\
& =-\frac{\log \operatorname{det} M^{\mathrm{free}}\left(T, \mu_{I}\right)}{V T^{3}} \\
& \approx C_{2} \frac{N_{f}}{2}\left(\frac{\mu_{I}}{T}\right)^{2}-C_{4} \frac{N_{f}}{4 \pi^{2}}\left(\frac{\mu_{I}}{T}\right)^{4},
\end{aligned}
$$

where $C_{2}$ and $C_{4}$ are fit coefficients. Table I summarizes the results.

The coefficients $C_{2}$ and $C_{4}$ approach their infinite volume expectation rather quickly. For the particular quark mass $\frac{m}{T}=0.2$ which we consider, the difference from the massless limit is smaller than the (fitting) errors, and thus,

\footnotetext{
${ }^{8}$ The $8^{3} \times 4$-data points are taken from the histogram method.
} 
TABLE I. The prediction for the free energy density based on the free massless gas model in the continuum at high temperature suffers from finite-size and cutoff effects. The correction terms $C_{2}$ and $C_{4}$ help to quantify the systematics. The functional form in Eq. (32) is sufficient, since the contribution of the additional term $C_{6}\left(\frac{\mu}{T}\right)^{6}$ is very small.

\begin{tabular}{lccc}
\hline \hline Lattice & $C_{2}$ & $C_{4}$ & {$\left[C_{6}\right]$} \\
\hline $4^{3} \times 4$ & $4.387(1)$ & $0.28(3)$ & {$[\cdots]$} \\
$6^{3} \times 4$ & $2.628(1)$ & $1.70(5)$ & {$[0.0081(1)]$} \\
$8^{3} \times 4$ & $2.315(1)$ & $2.25(5)$ & {$[0.0046(1)]$} \\
$10^{3} \times 4$ & $2.250(1)$ & $2.49(5)$ & {$[0.0030(1)]$} \\
$\infty^{3} \times 4$ & 2.25 & 2.6 & $\cdots$ \\
\hline \hline
\end{tabular}

TABLE II. The coefficients of the free energy density expansion for $\frac{T}{T_{c}} \sim 1.1$ come close to their Stefan-Boltzmann $(T \rightarrow \infty)$ value. There are two values for $b_{4}(T)$ : the first one is the result of the chi-square fit of Eq. (33); the second one ("periodic") makes use of a periodicised Ansatz, see Eq. (34). The comparison of the two values gives some measure of the systematic error.

\begin{tabular}{lccc}
\hline \hline$T \sim 1.1 T_{c}$ & $b_{2}(T)$ & $b_{4}(T)$ & $b_{4}(T)$ (periodic) \\
\hline $4^{3} \times 4$ & $0.29(1)$ & $32(4)$ & $7(1)$ \\
$6^{3} \times 4$ & $0.71(1)$ & $4.1(4)$ & $0.2(7)$ \\
$8^{3} \times 4$ & $0.90(2)$ & $3.6(8)$ & $1.4(4)$ \\
SB limit $(T \rightarrow \infty)$ & 1 & 1 & 1 \\
\hline \hline
\end{tabular}

results are not presented explicitly. Note that we have an additional column $\left[C_{6}\right]$ : we have added the term $C_{6}\left(\frac{\mu}{T}\right)^{6}$ to the Ansatz equation (32). The coefficient $C_{6}$ is very small and leaves $C_{2}$ and $C_{4}$ unchanged within the errors.

In the end, we consider the volume-dependent lattice corrections $C_{2}$ and $C_{4}$ and measure the deviation from this free gas model by two parameters $b_{2}(T)$ and $b_{4}(T)$. The Ansatz to describe our results in Fig. 5 is thus

$$
\frac{\Delta F\left(T, \mu_{I}\right)}{V T^{4}}=b_{2}(T) C_{2} \frac{N_{f}}{2}\left(\frac{\mu_{I}}{T}\right)^{2}-b_{4}(T) C_{4} \frac{N_{f}}{4 \pi^{2}}\left(\frac{\mu_{I}}{T}\right)^{4} .
$$

We observe that the leading term approaches the StefanBoltzmann limit rather fast upon increasing the volume, see Fig. 5 and Table II. This is somewhat surprising since this coincidence with the Stefan-Boltzmann law will occur only at $T \rightarrow \infty$. Deviations at $T \sim 1.1 T_{c}$ should persist even in the thermodynamic limit, reflecting the interactions of the quarks. ${ }^{9}$ The reduction of $b_{2}(T)$ from 1 is consistent with leading perturbative corrections [17]. The value we obtain is consistent with that measured in Ref. [20]. The simple prediction of the free massless quark gas model works better than expected. Thus, the relevant degrees of freedom at high temperature are very light quarks, which is also visible in the strong dependency of the free energy density on the imaginary chemical potential, hence $m_{q} \ll$ $\mu_{I} \sim T_{c} \approx 160 \mathrm{MeV}$.

The coefficient $b_{4}(T)$ in Table II suffers from systematic fitting errors. One source is the fitting range: our Ansatz equation (33) does not reflect the $\frac{2 \pi}{3}$-periodicity of $\frac{\mu_{I}}{T}$, therefore we are allowed to fit small $\frac{\mu_{I}}{T}$ only. In this regime, the quartic term is subleading and hard to quantify. An estimate of the systematic fitting error can be obtained by varying the fitting range (not explicitly tabulated). Another source is the fitting Ansatz: we could add the next-order term $\left(\frac{\mu_{I}}{T}\right)^{6}$, which changes $C_{4}$ by a few percent, or make the Ansatz periodic via

$$
\frac{\Delta F_{\mathrm{per}}\left(T, \mu_{I}\right)}{V T^{4}}=-\frac{1}{V T^{3}} \log \frac{Z_{\mathrm{per}}\left(T, \mu_{I}\right)}{Z_{\mathrm{per}}(T, 0)}
$$

with

$$
Z_{\mathrm{per}}\left(T, \mu_{I}\right)=\sum_{k=-\infty}^{\infty} \exp \left(-V T^{3}\left(b_{2}(T) C_{2} \frac{N_{f}}{2}\left(\frac{\mu_{I}}{T}+\frac{2 \pi k}{3}\right)^{2}-b_{4}(T) C_{4} \frac{N_{f}}{4 \pi^{2}}\left(\frac{\mu_{I}}{T}+\frac{2 \pi k}{3}\right)^{4}\right)\right)
$$

However, we must truncate (in practice, at $k= \pm 1$ ) the sum over all sectors to preserve convergence, because of the sign of the $C_{4}$ contribution. In conclusion, we cannot determine $b_{4}(T)$ accurately. Nevertheless, it is remarkable how well the free quark gas model describes our results. On an $8^{3} \times 4$ lattice, the deviation is only about $10 \%$. By using a canonical approach to simulate finite density QCD [3], we can obtain more accurate results, to be presented in a

\footnotetext{
${ }^{9}$ It has been shown already that the free energy of the gluon sector deviates from the Stefan-Boltzmann value $\frac{8 \pi^{2}}{45}$ by about $15 \%$ [19] even at $\frac{T}{T_{c}} \sim 5$ (and more for lower temperatures). It thus would be natural to observe deviations at finite temperature also in the quark sector.
}

follow-up publication. In particular, we can show that $b_{4}\left(T=1.1 T_{c}\right)=1.94(6)[2]$.

\section{FINITE-SIZE EFFECTS ON THE PHASE TRANSITION}

The phase transition is signaled by the peak in the susceptibility of the chiral condensate $\langle\bar{\psi} \psi\rangle$ (chiral transition) or in the specific heat (deconfinement transition). In Fig. 6, we compare the grand-canonical versus the canonical ensemble. Although there is no theoretical argument that the two transitions should occur together, we observe that within our accuracy they do.

On the $4^{3} \times 4$ lattice, a slight shift in the pseudocritical $\beta_{c}$ can be observed between the grand-canonical and the 

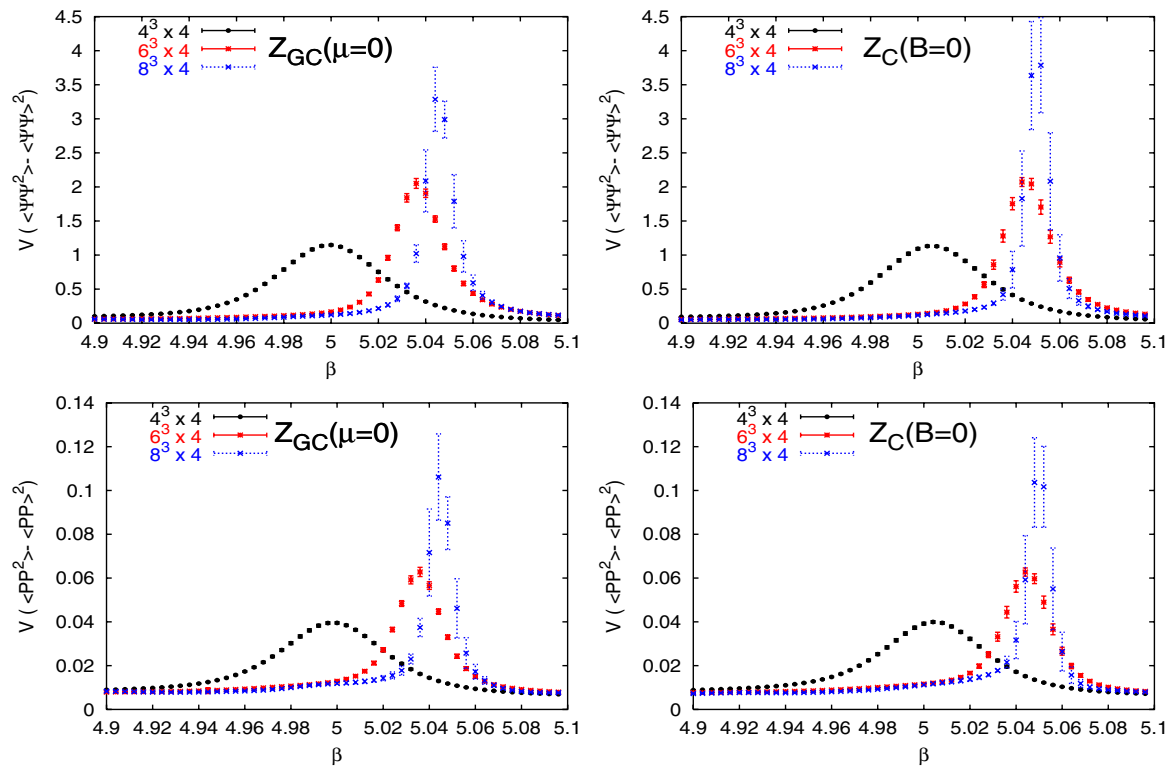

FIG. 6 (color online). Susceptibility of $\bar{\psi} \psi$ (top) and specific heat, given by the susceptibility of the plaquette (bottom), versus $\beta$ for increasing volumes, in the grand-canonical (left) and canonical (right) ensembles. Even for the smallest, $4^{4}$, lattice, differences between the two ensembles are barely visible.
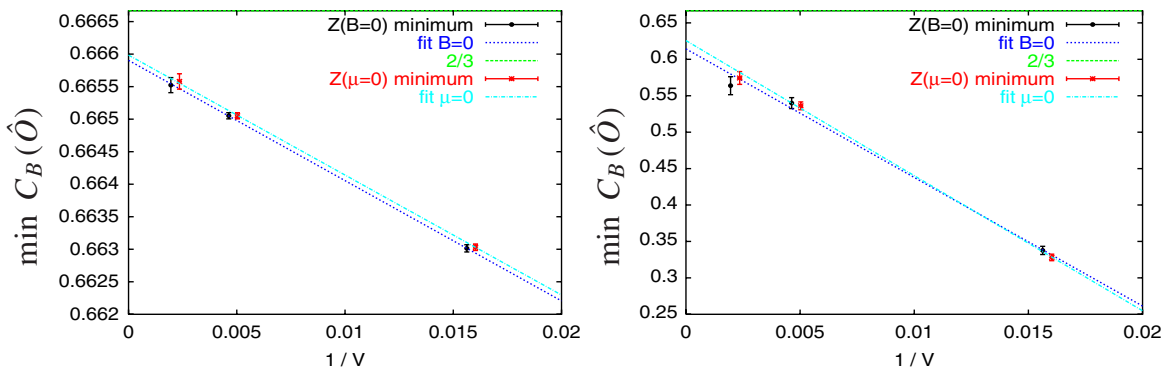

FIG. 7 (color online). Binder cumulant minimum versus inverse volume for both ensembles (slightly shifted in the $x$-axis to enhance visibility). Left: $\hat{O}=$ plaquette, right: $\hat{O}=$ chiral condensate. The thermodynamic extrapolation does not reach $\frac{2}{3}$ (the upper left corner of the figure), indicating a first-order transition. Finite-size effects, reflected in the $1 / V$ slope, are equivalent for both ensembles.

canonical results. It disappears for larger volumes. The small deviation is caused by contributions from $B \neq 0$ sectors, which are present in the $\mu=0$ ensemble. However they are suppressed by a factor $\sim e^{-B m_{B} / T} \ll 1$, where $m_{B}$ is the mass of a baryon. In terms of the baryon density $\rho$, we recognize the exponential suppression in the volume since $e^{-B m_{B} / T}=e^{-V \rho m_{B} / T}$. Thus, we verify once more that the zero chemical potential ensemble is equivalent to the zero baryon density ensemble in the thermodynamic limit.

Note that the nonzero triality sectors have zero partition function and do not contribute. They do not affect observables studied in this section, which are insensitive to the center symmetry.

In quenched simulations, a $Z_{3}$-symmetrization of the Polyakov loop is sometimes enforced by hand, which is accompanied by reduced finite-size effects [21]. Therefore, we might expect to observe a similar reduction in the canonical formalism as well compared to the grandcanonical one. To compare the finite-size effects in the two ensembles, we analyze the minimum of the Binder cumulant [22]

$$
C_{B}(\hat{O})=1-\frac{1}{3} \frac{\left\langle\hat{O}^{4}\right\rangle}{\left\langle\hat{O}^{2}\right\rangle^{2}}
$$

versus the inverse volume $1 / V$ (see Fig. 7). For both the plaquette and the chiral condensate, the thermodynamic (linear) extrapolation does not tend to $\frac{2}{3}$-indicative of a first-order phase transition, ${ }^{10}$ confirming the finding in the literature [23] for our quark masses. However, for each

\footnotetext{
${ }^{10}$ In the case of a second-order transition or a crossover, $\left\langle\hat{O}^{4}\right\rangle$ is equal to $\left\langle\hat{O}^{2}\right\rangle^{2}$ up to finite-size corrections [22]. Thus, $C_{B}(\hat{O}) \rightarrow$ $\frac{2}{3}$ in the thermodynamic limit. In the case of a first-order transition, the double peak structure of the distribution of the measurements causes a nontrivial value of the Binder cumulant.
} 
volume, the measured cumulant values agree between the two ensembles within statistical errors, indicating equivalent finite-size effects.

\section{CONCLUSIONS}

For all densities, volumes, and (finite) temperatures, the Polyakov loop expectation value is nonzero in the grandcanonical ensemble Eq. (1), and zero in the equivalent canonical ensemble Eq. (2). This Polyakov loop paradox has to be considered an artifact of keeping, in the grandcanonical ensemble, sectors with quark numbers not multiple of three. These canonical sectors, the so-called nonzero triality sectors, have zero partition function. Thus, the nonvanishing expectation value $\langle\mathrm{Pol}\rangle_{Z_{\mathrm{GC}}(T, \mu)}$ in the common grand-canonical formulation of QCD at finite temperature and density, Eq. (1), is irrelevant for thermodynamic properties. The physically meaningful Polyakov loop correlator $\left\langle\operatorname{Pol}(0) \operatorname{Pol}(x)^{\dagger}\right\rangle$ behaves in the same way in both ensembles.

Because of quantum and thermal fluctuations, $\left\langle\operatorname{Pol}(0) \operatorname{Pol}(x)^{\dagger}\right\rangle$ tends to a nonzero value when $|x| \rightarrow \infty$. On the other hand, $\langle\mathrm{Pol}\rangle=0$ in the canonical ensemble. Thus, the clustering property is violated, which shows that the center symmetry is spontaneously broken in the canonical ensemble, rather than explicitly broken by the fermion determinant as in the usual grand-canonical ensemble.

Furthermore, an explicitly center-symmetric grandcanonical partition function $Z_{\mathrm{GC}}(T, \mu)$, Eq. (8), can be constructed from the canonical partition functions, where the contributions of nonzero triality states are projected out. This partition function will give identical expectation values to the usual $Z_{\mathrm{GC}}(T, \mu)$, apart from a vanishing expectation value for the Polyakov loop. Therefore, the nonzero triality states can be included or excluded: the thermodynamic properties of the theory are unchanged.

We have shown this explicitly by comparing the grandcanonical ensemble at $\mu=0$ with the canonical ensemble $B=0$. Numerically, we have established the equivalence of $Z_{\mathrm{GC}}(\mu=0)$ and $Z_{C}(B=0)$ in the thermodynamic limit by measuring the free energy density as a function of $\mu_{I}$, using the histogram of the imaginary chemical potential distribution. ${ }^{11}$ For all temperatures, we observe a minimum at $\frac{\mu_{I}}{T}=0$. At low temperature, the free energy density of the confined phase can be rather well described by the hadron resonance gas, see Eq. (30). We thus have a simple way to determine the sum of resonances $f(T)$. At high temperature, a slightly modified free gas Ansatz, see
Eq. (33), allows to account for all data points in the quark-gluon plasma phase. We determine the finite-size and cutoff correction terms $C_{2}$ and $C_{4}$ by calculating the free energy of the free fermion gas on the lattice and find agreement with the literature for $V \rightarrow \infty$. By construction, the interaction coefficients $b_{2}(T)$ and $b_{4}(T)$ tend to 1 for high enough temperatures, reproducing the StefanBoltzmann law. Just above $T_{c}$, the deviation from 1 in the leading coefficient is about $30 \%$ on a $6^{3} \times 4$ lattice; on an $8^{3} \times 4$ lattice, this deviation is about $10 \%$ only. This near-agreement with a noninteracting gas is unexpected at such comparatively low temperatures.

The approach to the thermodynamic limit is very similar in the canonical $(B=0)$ and grand-canonical $(\mu=0)$ ensembles. The susceptibility of the chiral condensate, or the specific heat, indicate the same pseudocritical temperature already on small volumes. A small shift, caused by contributions from nonzero baryon sectors, is visible only on the $4^{3} \times 4$ lattice.

The zero-density canonical formulation requires a center-symmetric simulation of QCD, which can be achieved very simply with negligible computer overhead, by adding to the standard algorithm a single degree of freedom $\mu_{I}$ updated by Metropolis.

We hope to have fully clarified the (un)importance of nonzero triality states, and thus, to have put to rest longstanding speculations. Further connections between the grand-canonical and the canonical formalisms in the context of nonzero chemical potential/density will be the subject of a forthcoming paper [2].

\section{ACKNOWLEDGMENTS}

We are grateful to O. Jahn, K. Kajantie, F. Karsch, M. P. Lombardo, O. Philipsen, K. Rummukainen, B. Svetitsky, T. Takaishi, D. Toublan, and L. G. Yaffe for fruitful discussions and advice.

\section{APPENDIX: STOCHASTIC ESTIMATOR}

A ratio of determinants can be estimated using a single Gaussian complex vector:

$$
\begin{aligned}
\frac{\operatorname{det}^{N_{f}}\left(\not D\left(\mu_{I}^{\prime}\right)+m\right)}{\operatorname{det}^{N_{f}}\left(\not D\left(\mu_{I}\right)+m\right)} & =\frac{\operatorname{det}^{N_{f}} M\left(\mu_{I}^{\prime}\right)}{\operatorname{det}^{N_{f}} M\left(\mu_{I}\right)} \\
& =\frac{\int d \phi^{\dagger} d \phi e^{-\phi^{\dagger}\left(1 / M^{N_{f}}\left(\mu_{I}^{\prime}\right)\right) \phi}}{\int d \phi^{\dagger} d \phi e^{-\phi^{\dagger}\left(1 / M^{N_{f}}\left(\mu_{I}\right)\right) \phi}}
\end{aligned}
$$

\footnotetext{
${ }^{11}$ Remember that our numerical approach treats $\mu_{I}$ as a dynamical degree of freedom.
} 
where we have substituted $\phi=M^{N_{f} / 2}\left(\mu_{I}\right) \eta$. Note that in the above notation, the Jacobian $\left|J\left(\phi, \eta, \mu_{I}\right)\right|$ is $\operatorname{det} M^{N_{f}}\left(\mu_{I}\right)$, which is independent of $\eta$ and cancels out in the ratio:

$$
\begin{gathered}
\frac{\operatorname{det}^{N_{f}}\left(\not \not\left(\mu_{I}^{\prime}\right)+m\right)}{\operatorname{det}^{N_{f}}\left(\not D\left(\mu_{I}\right)+m\right)}=\frac{\int d \eta^{\dagger} d \eta e^{-\left|M^{-N_{f} / 2}\left(\mu_{I}^{\prime}\right) M^{N_{f} / 2}\left(\mu_{I}\right) \eta\right|^{2}} e^{-|\eta|^{2}+|\eta|^{2}}}{\int d \eta^{\dagger} d \eta e^{-|\eta|^{2}}} \\
=\left\langle e^{-\left|M^{-N_{f} / 2}\left(\mu_{I}^{\prime}\right) M^{N_{f} / 2}\left(\mu_{I}\right) \eta\right|^{2}+|\eta|^{2}}\right\rangle_{\eta} .
\end{gathered}
$$

$\langle\cdot\rangle_{\eta}$ tells us that $\eta$ has to be sampled with the distribution $\int d \eta^{\dagger} d \eta e^{-|\eta|^{2}}$.

[1] Z. Fodor and S. D. Katz, Phys. Lett. B 534, 87 (2002); C. R. Allton et al., Phys. Rev. D 66, 074507 (2002); P. de Forcrand and O. Philipsen, Nucl. Phys. B642, 290 (2002); M. D'Elia and M. P. Lombardo, Phys. Rev. D 67, 014505 (2003).

[2] S. Kratochvila and P. de Forcrand, Nucl. Phys. B, Proc. Suppl. 140, 514 (2005); A. Alexandru, M. Faber, I. Horvath, and K. F. Liu, Nucl. Phys. B, Proc. Suppl. 140, 517 (2005); Phys. Rev. D 72, 114513 (2005).

[3] S. Kratochvila and P. de Forcrand, Proc. Sci., LAT2005 (2006) 167 [hep-lat/0509143]; Nucl. Phys. B, Proc. Suppl. 153, 62 (2006).

[4] J. Engels, O. Kaczmarek, F. Karsch, and E. Laermann, Nucl. Phys. B558, 307 (1999).

[5] V. Azcoiti and A. Galante, Phys. Lett. B 444, 421 (1998).

[6] S. Kratochvila and P. de Forcrand, Prog. Theor. Phys. Suppl. 153, 330 (2004); Nucl. Phys. B, Proc. Suppl. 129, 533 (2004).

[7] M. Oleszczuk and J. Polonyi, Ann. Phys. (N.Y.) 227, 76 (1993); M. Oleszczuk, Nucl. Phys. B, Proc. Suppl. 39, 471 (1995); O. Borisenko, M. Faber, and G. Zinovev, Nucl. Phys. B444, 563 (1995); K. Fukushima, Ann. Phys. (N.Y.) 304, 72 (2003).

[8] R. Hagedorn and J. Rafelski, CERN Report No. CERNTH-2947, 1980 (unpublished); CERN Report No. CERNTH-2969, 1980 (unpublished).
[9] A. Roberge and N. Weiss, Nucl. Phys. B275, 734 (1986).

[10] S. Duane, A. D. Kennedy, B. J. Pendleton, and D. Roweth, Phys. Lett. B 195, 216 (1987).

[11] M. G. Alford, A. Kapustin, and F. Wilczek, Phys. Rev. D 59, 054502 (1999).

[12] B. A. Berg and T. Neuhaus, Phys. Rev. Lett. 68, 9 (1992).

[13] M.P. Lombardo (private communication).

[14] A. M. Ferrenberg and R. H. Swendsen, Phys. Rev. Lett. 61, 2635 (1988); 63, 1195 (1989).

[15] C. R. Allton et al., Phys. Rev. D 66, 074507 (2002).

[16] C. R. Allton et al., Phys. Rev. D 68, 014507 (2003).

[17] C. R. Allton et al., Phys. Rev. D 71, 054508 (2005).

[18] D. Toublan (private communication).

[19] G. Boyd, J. Engels, F. Karsch, E. Laermann, C. Legeland, M. Lutgemeier, and B. Petersson, Nucl. Phys. B469, 419 (1996).

[20] M. D'Elia and M. P. Lombardo, Phys. Rev. D 70, 074509 (2004); M. D'Elia, F. Di Renzo, and M. P. Lombardo, AIP Conf. Proc. No. 806 (AIP, New York, 2006), p. 245.

[21] G. Martinelli, G. Parisi, R. Petronzio, and F. Rapuano, Phys. Lett. B 122, 283 (1983).

[22] K. Binder, Z. Phys. B 43, 119 (1981).

[23] M. Fukugita and A. Ukawa, Phys. Rev. Lett. 57, 503 (1986). 